\title{
Effet d'un environnement informatique pour l'apprentissage humain sur la motivation des élèves à faire des mathématiques : caractéristiques des élèves et style de l'enseignant
}

Stéphanie Reyssier

ISPEF - Université Lyon 2

Stéphane Simonian

ISPEF - Université Lyon 2

\section{Résumé}

Cette recherche s'intéresse à l'effet d'un environnement informatique pour l'apprentissage humain (EIAH), coconçu avec des enseignants, sur la variation de la motivation des élèves à faire des mathématiques en fonction de leur sexe, de l'appartenance socioéconomique, du niveau de motivation initiale et du style motivationnel de l'enseignant lors de l'usage de cet EIAH en classe. Les principaux résultats, obtenus auprès de 163 élèves, en mesurant leur motivation en prétest et posttest via l'échelle de Vallerand (Vallerand et al., 1989), montrent

Canadian Journal of Education / Revue canadienne de l'éducation 44:1 (2021)

C2021 Canadian Society for the Study of Education/ Société canadienne pour l'étude de l'éducation 
un effet positif de l'EIAH en fonction de certaines caractéristiques des élèves, notamment pour ceux étant initialement les plus démotivés à faire des mathématiques, et inversement. Cet effet se vérifie particulièrement lorsque l'enseignant favorise l'autonomie des élèves.

Mots-clés : EIAH, mathématiques, motivation autonome, modalités pédagogiques

\begin{abstract}
This research deals with the effect of a computer-based learning environment (CBLE), co-designed with teachers, on the variation of students' motivation to do mathematics, according to their gender, socioeconomic background, initial motivation level, and the teacher's motivational style, while using this CBLE in the classroom. The main results, obtained from 163 students by using the Vallerand academic motivation scale (Vallerand et al., 1989) pre-test and post-test, highlight the positive effect of the CBLE, according to certain student characteristics, especially for those students who were initially the least motivated to do mathematics and vice versa. This effect is particularly true when the teacher promotes students' autonomy.

Keywords: CBLE, mathematics, autonomous motivation, teaching methods

\section{Remerciements}

Nous remercions les enseignants et élèves ayant participé à cette recherche dans le cadre du projet LudiMoodle, développé par l'Université de Lyon et soutenu par l'État, dans le cadre du volet e-FRAN du Programme d'investissement d'avenir, opéré par la Caisse des Dépôts.
\end{abstract}




\section{Introduction}

La présente étude s'inscrit dans la réponse à un appel à projet e-FRAN, du Programme d'investissements d'avenir (PIA) français, dans l'objectif général de soutenir et d'augmenter les performances scolaires des élèves, considérant qu'un environnement informatique pour l'apprentissage humain (EIAH) bien conçu favorise l'autonomie et la personnalisation des apprentissages (Balanskat et al., 2006), mais aussi que la motivation varie selon des caractéristiques individuelles (Garon-Carrier et al., 2016), 1'appartenance socioéconomique (Chouinard et al., 2010) et le niveau de motivation initiale (Lavoué et al., 2018). C'est dans ce contexte que s'inscrit le présent projet intitulé « LudiMoodle », développé par l'Université de Lyon ${ }^{1}$. Il vise à favoriser la motivation d'élèves de $4 \mathrm{e}$ année ${ }^{2}$ en mathématiques, et plus précisément en calcul littéral, notamment en proposant à terme un EIAH ludifié ${ }^{3}$ (Deterding et al., 2011). Cette recherche se situe à la première étape du projet dont l'objectif est double : évaluer, du point de vue de la théorie de l'autodétermination ([TAD] Ryan et Deci, 2000), la pertinence de l'EIAH en termes de gain motivationnel pour les élèves en tentant compte : 1) des caractéristiques des élèves et 2) de la manière dont l'enseignant appréhende l'EIAH et l'intègre à la situation d'apprentissage. Ce n'est qu'à partir de ces données que la ludification pourra être envisagée, afin de l'adapter aux utilisateurs et à la situation d'apprentissage (2e étape du projet).

1 Ce projet regroupe deux laboratoires de recherche : le laboratoire Éducation Cultures Politiques (Lyon 2) et le Laboratoire d'Informatique en Images et Systèmes d'Information (Lyon 1), qui se sont associés au Pôle d'Accompagnement à la Pédagogie Numérique (Lyon 3), spécialisé en ingénierie pédagogique, et à l'entreprise Edunao, spécialiste de la plateforme Moodle, pour développer des ressources en mathématiques ludifiées en collaboration avec des enseignants.

2 La 4e année du système scolaire français correspond au 8e grade du système canadien ou au secondaire $2 \mathrm{du}$ système québécois (13-14 ans). Pour une comparaison des différents systèmes scolaires, voir http://fisna.org/ scolarite/lorganisation-de-la-scolarite/comparaison-avec-les-usa-et-le-canada/

3 EIAH ludifié : EIAH enrichi par des éléments issus du jeu tels qu'un avatar, des points, des badges ou une minuterie. 


\section{Problématique}

\section{Théorie de l'autodétermination et motivations}

En 1975, Deci suppose que la motivation intrinsèque peut être influencée par la perception du locus de causalité (DeCharms, 1968 ; Heider, 1982) qui correspond à la perception qu'un individu a du degré d'intentionnalité de ses actes ; intentionnalité pouvant être intrapersonnelle (faisant référence à « l'auto ») et/ou interpersonnelle (Ryan et Connell, 1989 ; Vallerand et Reid, 1984). Plus les causes interpersonnelles externes seraient internalisées, plus l'autorégulation interne serait importante. Deci et Ryan (1985) proposent quant à eux une taxonomie quadripartite de la motivation extrinsèque afin de tenir compte des degrés potentiellement différents d'intériorisation dans le soi. Ils distinguent ainsi les motivations extrinsèques à la régulation externe (agir dans le but d'obtenir une récompense ou d'éviter une punition), à la régulation introjectée (agir pour éviter la honte ou l'atteinte de l'estime de soi), à la régulation identifiée (agir dans le but d'atteindre un objectif précis) et à la régulation intégrée (agir en accord avec ses valeurs). Cette dernière, liée au degré de maturation psychique de l'individu, serait observable principalement chez l'adulte. C'est pourquoi Vallerand et Blais (1987), pour répondre aux suggestions de Deci de différencier différents types de motivations intrinsèques et extrinsèques, vont proposer une taxonomie tripartite de la motivation intrinsèque, distinguant ainsi les motivations intrinsèques à la stimulation (liée au plaisir de réaliser une activité), à l'accomplissement (liée aux besoins de relever des défis et de se sentir compétent) ou à la connaissance (liée au désir d'apprendre). Vallerand (1997) propose d'utiliser le terme « amotivation » comme l'absence d'intention d'émettre un comportement (la raison étant que l'individu ne perçoit pas de bonnes raisons de le faire) afin de tenir compte de toutes les situations où les individus ne semblent pas motivés à agir. Vallerand et al. (1989) proposent alors une échelle de la motivation en éducation, ayant une cohérence interne satisfaisante, destinée à des élèves du secondaire. Cette échelle intègre les trois formes de motivations intrinsèques, les quatre formes de motivations extrinsèques, mais aussi l'amotivation (cf. tableau 1). L'amotivation correspond à « l'orientation-impersonnelle » que Deci et Ryan (1985) associent à un 
locus de contrôle externe. Ces différents types de motivations extrinsèques, intrinsèques et l'amotivation constituent un continuum d'autodétermination (cf. tableau 1).

\section{Tableau 1}

Continuum d'autodétermination d'après Deci et Ryan (2000)

\begin{tabular}{ccccccc}
\hline $\begin{array}{c}\text { Type de } \\
\text { motivation }\end{array}$ & Amotivation & & Motivation extrinsèque & & $\begin{array}{c}\text { Motivation } \\
\text { intrinsèque }\end{array}$ \\
\hline $\begin{array}{c}\text { Type de } \\
\text { régulation }\end{array}$ & $\begin{array}{c}\text { Pas de } \\
\text { régulation }\end{array}$ & $\begin{array}{c}\text { Régulation } \\
\text { externe }\end{array}$ & $\begin{array}{c}\text { Régulation } \\
\text { introjectée }\end{array}$ & $\begin{array}{c}\text { Régulation } \\
\text { identifiée }\end{array}$ & $\begin{array}{c}\text { Régulation } \\
\text { intégrée }\end{array}$ & $\begin{array}{c}\text { Connaissance } \\
\text { Accomplisse- } \\
\text { ment } \\
\text { Stimulation }\end{array}$ \\
$\begin{array}{c}\text { Locus de } \\
\text { causalité }\end{array}$ & Impersonnelle & Externe & $\begin{array}{c}\text { Partiellement } \\
\text { externe }\end{array}$ & $\begin{array}{c}\text { Partiellement } \\
\text { interne }\end{array}$ & Interne & Interne \\
$\begin{array}{c}\text { Comporte- } \\
\text { ment }\end{array}$ & Non déterminé & & & & & Déterminé \\
\hline
\end{tabular}

\section{Théorie de l'autodétermination et besoin d'autonomie}

De manière générale, les études sur la motivation (Deci et Ryan, 2000) montrent des effets différents en fonction du degré d'autonomie laissé à l'élève : plus l'autonomie serait favorisée, plus la motivation intrinsèque augmenterait (Gillet et al., 2012 ; Grolnick et al., 1991). Ceci est un enjeu important dans le secteur éducatif étant donné que la motivation intrinsèque serait positivement corrélée aux performances scolaires de l'élève (Garon-Carrier et al., 2016). Cependant, la motivation est aussi « située » (Boekaerts, 2001 ; Bouffard et al., 2004), dépendante de l'objet de savoir étudié, de caractéristiques individuelles, de la situation d'apprentissage et des artefacts utilisés (Deterding et al., 2011).

La TAD (Ryan et Deci, 2000) est une théorie de la motivation qui cherche à comprendre les raisons qui poussent les individus à agir, à se comporter d'une façon plutôt qu'une autre, et ce, dans de nombreux domaines, que ce soit en éducation physique et sportive, en santé, au travail ou dans le contexte scolaire. Elle s'inscrit dans une perspective organismique de la motivation, reposant sur le concept d'internalisation des contraintes extérieures dans le soi. Cette internalisation serait un processus proactif par 
lequel les individus transformeraient des régulations externes en régulations internes (Deci et al., 1991), afin de s'assurer un fonctionnement harmonieux dans le monde social auquel ils appartiennent (Blais et al., 1990 ; Deci et Ryan, 2000 ; Reis et al., 2000). Ainsi, plus les contraintes externes seraient internalisées, plus l'individu serait autodéterminé à agir. Cette intériorisation des contraintes externes dans le soi (Ryan et Deci, 2002) serait notamment favorisée par la satisfaction du besoin d'autonomie ${ }^{4}$, qui influencerait positivement la progression de motivations autonomes (motivations intrinsèques, régulations identifiée et intégrée) et, plus spécifiquement, la motivation intrinsèque, directement liée au plaisir de réaliser une activité (Deci et Ryan, 1980 ; Ryan et Grolnick, 1986).

L'autodétermination, en tant que motivation autonome, ne semble pas pouvoir se généraliser à tous les types d'individus, puisqu'elle dépendrait du contexte et de certaines caractéristiques individuelles. Il semblerait en effet que l'âge et la maturité psychologique (Chandler et Connell, 1987) en soient prépondérants : seuls les adultes auraient une régulation intégrée leur permettant d'agir en accord avec leurs propres valeurs (Vallerand et al., 1989), les adolescents connaissant un déclin des formes autodéterminées et contrôlées de leur motivation entre 9 et 15 ans, au profit d'une plus grande amotivation (Gillet et al., 2012 ; Vallerand, 1997).

D'autres variables, tels le sexe ou l'appartenance socioéconomique, seraient à considérer. Une recherche de Garron-Carrier et al. (2016) montre que les garçons auraient une motivation intrinsèque supérieure à celle des filles, et ce, quel que soit l'âge considéré. Souchal et Toczek (2010) constatent des performances plus élevées des élèves de milieux favorisés, ce qu'elles attribuent aux buts de maîtrise qu'ils se fixent, contrairement aux élèves de milieux défavorisés qui poursuivraient plutôt des buts de performance. Elles nuancent cependant ces résultats, constatant des effets différents suivant le contexte d'apprentissage. Pour Chouinard et al. (2010), les élèves de milieux défavorisés percevraient moins l'utilité de l'école que les autres ; ceux de milieux favorisés auraient une attitude plus positive et autodéterminée (Damon et Lerner, 2006). Ainsi, nous comprenons l'importance de tenir compte des caractéristiques de l'élève, mais aussi du contexte d'apprentissage dans le but de favoriser leur motivation (Legrain, 2003).

4 L'intériorisation des contraintes externes dépend aussi de la réponse à deux autres besoins psychologiques : les besoins de compétence et d'appartenance à un groupe. 


\section{Favoriser la motivation autonome en contexte scolaire}

Dans le secteur éducatif et formatif, la relation entre la motivation et la réussite suppose de tenir compte des caractéristiques des élèves (Legrain, 2003). Il est notamment constaté un effet « enseignant » marqué par des différences de méthodes pédagogiques et de degré d'autonomie laissé aux élèves (Careau et Fournier, 2002 ; Viau, 2009). En effet, la motivation dépend fortement des agents sociaux qui permettent de fournir un soutien à l'autonomie, particulièrement les enseignants, dont le soutien influence directement la motivation intrinsèque et la motivation externe à la régulation introjectée (Gillet et al., 2012). En d'autres termes, la motivation de l'élève dépend de la manière dont l'enseignant favorise et accompagne l'autonomie de l'élève (Deci et Ryan, 2000) en lui proposant des exercices variés et en tenant compte des objectifs des élèves (Supper et al., 2019).

Si la théorie de l'autodétermination vise à mieux comprendre les processus cognitifs, affectifs et comportementaux, un des enjeux dans le secteur éducatif concerne la manière dont un enseignant peut améliorer la motivation des élèves (Benita et al., 2017 ; Careau et Fournier, 2002 ; Ciani et al., 2011 ; Gillet et al., 2012 ; Vansteenkiste et al., 2010 ; Viau, 2009) selon la manière de conduire les activités réalisées (Grolnick et Ryan, 1987). Contrairement aux situations d'apprentissage trop contrôlées, soumises à des évaluations systématiques (Harackiewicz et al., 1984), en particulier celles avec l'obligation de finir la tâche ou de performance (Amabile et al., 1976 ; Deci et al., 2001), le fait de laisser l'apprenant libre d'agir tout en étant guidé et de favoriser un climat motivationnel bienveillant sont déterminants sur les motivations autonomes (Sarrazin et al., 2006) : un climat de maîtrise valorisant les efforts, le travail et les progrès des élèves favoriserait leur autonomie et les apprentissages profonds, contrairement à un climat de compétition (Deci et al., 1981 ; Ryan et Grolnick, 1986), qui incite plutôt à la comparaison sociale et aux apprentissages de surface (Cosnefroy, 2010 ; Galand et al., 2006 ; Ryan et al., 1983). Le style de l'enseignant - contrôlant versus non contrôlant - serait déterminant, car il influerait sur la perception d'autonomie des élèves (Chirkov et Ryan, 2001). Selon Roth et al. (2007), plus l'enseignant serait contrôlant, moins il soutiendrait l'autonomie de ses élèves. Pour Reeve (2009), un enseignant contrôlant aurait tendance à monopoliser le matériel pédagogique, ne laisserait pas la possibilité aux élèves de réfléchir et de répondre aux problèmes posés et menacerait de punition. À 
l'inverse, un enseignant non contrôlant serait à l'écoute de ses élèves, leur permettrait de faire des choix, favoriserait les rétroactions positives et les temps de travail en autonomie. Il augmenterait ainsi leur processus d'autorégulation et favoriserait davantage leur motivation intrinsèque, contrairement à un enseignant contrôlant qui donnerait des directives « rigides ». Le degré de contrôle concernerait aussi le positionnement et les déplacements de l'enseignant dans la classe, sa manière de répondre aux difficultés des élèves et de les soutenir (Viau, 2009).

\section{EIAH et autonomie}

Lorsque la situation d'apprentissage est médiée et médiatisée par un EIAH (Rabardel, 1995 ; Tchounikine et Tricot, 2011), l'environnement comprend la spécificité de cet EIAH, son contexte d'application et le comportement des acteurs (enseignants et élèves). L'environnement est compris au sens large comme un environnement socioculturel où le savoir circule et se construit entre des acteurs médiés par un EIAH, mais aussi entre les acteurs et l'EIAH.

Un EIAH est un programme destiné à être utilisé par des apprenants impliqués dans une situation d'enseignement-apprentissage et accompagnés dans cet apprentissage. Un EIAH peut jouer plusieurs rôles non mutuellement exclusifs : présenter et structurer l'information, favoriser la communication, traiter de l'information. C'est dans ces premier et troisième rôles que s'inscrit cette étude, puisque l'EIAH développé est un système à base de connaissances où les notions sont présentées de manière progressive pour résoudre des exercices, à travers et sous son contrôle, avec des élèves. Ce type d'EIAH laisse une place importante au style de l'enseignant lorsqu'on prévoit en faire l'utilisation en classe. Ce style d'enseignement se caractérise par l'action singulière de l'enseignant en situation, y compris son usage des instruments techniques à disposition (Clot, 2008). Il dépendrait des possibilités que perçoit l'enseignant de cet EIAH (Garrett, 2002 ; Hassenzahl, 2010 ; Norman, 1988), des modalités qu'il met en œuvre, et de la manière dont il s'adapte plus ou moins en situation. Il influencerait le degré d'autonomie des élèves, et donc leur motivation autodéterminée (Black et Deci, 2000 ; Miserandino, 1996). La relation entre l'enseignant et l'EIAH est donc centrale en termes de « contrôle » pour favoriser l'autonomie : les « dispositifs flexibles et autonomisants » augmenteraient la liberté d'action des élèves et leur permettraient d'exercer un contrôle 
sur leurs apprentissages, notamment en faisant preuve de plus d'autodétermination et d'autorégulation (Jézégou, 2010, p. 85).

Les différentes méta-analyses sur l'effet des environnements numériques sur la motivation des élèves notent des résultats modérés et contrastés (Khalili et Shashaani, 1994 ; Michko, 2008 ; Tamim et al., 2011). Cependant, Leroux et al. (2017), dans une étude critique sur les méta-analyses, expliquent ces résultats par des différences de contexte, de durée d'expérimentations, mais aussi de caractéristiques individuelles des élèves : âge des élèves (Gillet et al., 2012), sexe (Fraillon et al., 2014 ; Leroux et al., 2017), appartenance socioculturelle (élève favorisé versus élève modeste : Cheung et Slavin, 2013 ; Jerrim, 2016). Morie et Goore (2019) montrent, plus spécifiquement dans le cadre d'un EIAH s'adaptant de façon dynamique aux progrès de l'élève, des résultats plus positifs chez les élèves ayant bénéficié d'un accompagnement de leur enseignant. Ce n'est donc pas l'implantation d'une technologie éducative qui améliore la motivation des élèves, mais la perception, puis l'usage qui en est fait par les enseignants (Karsenti et Fiévez, 2014 ; Puentedura, 2014), c'est-à-dire la manière dont il l'intègre à sa pratique. Ainsi, il est important de prendre en compte les pratiques pédagogiques des enseignants avec l'EIAH (Supper et al., 2019).

D'autres études portant sur les EIAH montrent de la démotivation à long terme (Koivisto et Hamari, 2014), ou des effets différents en termes de motivation autonome suivant l'âge des élèves (Gillet et al., 2012), leur sexe (Fraillon et al., 2014 ; Leroux et al., 2017), ou leur appartenance socioculturelle (élève favorisé versus élève modeste : Cheung et Slavin, 2013 ; Jerrim, 2016). Enfin, certains auteurs montrent des effets différents selon le type de motivation initiale (Hallifax et al., 2019 ; Lavoué et al., 2018).

Par conséquent, pour comprendre les effets d'un EIAH sur la motivation, il semble nécessaire de tenir compte du contexte d'apprentissage, de certaines caractéristiques des élèves et des pratiques enseignantes.

\section{Objectif et contribution}

L'objectif de cette recherche est de s'interroger sur les variables qui influent sur la variation de motivation des élèves, en considérant que l'autonomie occupe une position centrale dans les orientations causales (Deci et Ryan, 2000). L'individu ayant une orientation autonome serait libre de faire ses choix et de faire preuve de volition, ce 
qui lui permettrait de diriger ses buts et de réguler ses comportements en fonction du résultat à atteindre (Nix et al., 1999 ; Ryan et al., 1983). Ainsi, une des thèses centrales de la théorie de l'autodétermination est que la liberté d'action améliorerait la motivation intrinsèque (Vallerand, 2007 ; Zuckerman et al., 1978). La façon de présenter un message (comme une suggestion versus comme un ordre) aurait également une influence sur la motivation intrinsèque (Mageau et Vallerand, 2003 ; Pelletier et Vallerand, 1996) ; tout comme, dans notre contexte particulier, le style de l'enseignant — contrôlant versus non contrôlant (Deci et al., 1991 ; Ryan et Connell, 1989) —, la manière dont il interagit avec les élèves (Ryan, Grolnick, 1986), mais aussi, et de manière plus restrictive, la spécificité de l'EIAH conçu pour résoudre des problèmes en mathématiques : rétroaction, renforcement positif ou non, assistance à la conduite du raisonnement ou non, degré de liberté laissé à l'utilisateur. Cet ensemble de variables expliquerait des effets motivationnels différents selon les caractéristiques des élèves (sexe, appartenance socioculturelle, niveau de motivation initiale). C'est la raison pour laquelle cette étude contribue à évaluer, du point de vue de la théorie de l'autodétermination, la pertinence d'un EIAH dans une approche écologique, c'est-à-dire en considérant l'enseignant, l'EIAH et les élèves comme faisant partie d'un même environnement qui conditionne les potentialités que l'élève perçoit de se sentir autonome, et celles que l'enseignant perçoit de mettre ses élèves en posture d'autonomie.

\section{Méthodologie}

Un pré-test et un post-test ont permis de mesurer quantitativement la motivation des étudiants (Vallerand et al., 1989). Afin d'évaluer la situation d'apprentissage en termes de style d'enseignement « contrôlant », trois enseignants ont participé à des séances d'enseignement et à des entretiens d'explicitation d'actions filmés (Vermersch, 1994). Les résultats de ces observations, directement mis en lien avec ceux sur la motivation des élèves, ont permis d'étudier la congruence entre l'influence de la motivation en fonction des caractéristiques des élèves et du style de l'enseignant en situation réelle. Cependant, pour comprendre l'ensemble des résultats, il est nécessaire de connaître, en amont, les spécificités de l'EIAH coconçu avec ces trois mêmes enseignants de mathématiques. 


\section{Coconception de l'EIAH}

Pour favoriser son adaptation au contexte et aux pratiques éducatives existantes, cet EIAH a été coconçu avec des enseignants afin de s'assurer d'y intégrer certaines tâches effectuées par l'enseignant et permettre de médiatiser certaines de ses connaissances expertes. Cette coconception semble d'autant plus pertinente qu'un EIAH est un environnement informatique conjuguant une intention didactique et un environnement informatique qui modifie et réorganise la situation d'apprentissage. Il est en effet supposé que les outils qu'il mobilise, les potentialités qu'il perçoit et la façon dont il s'en sert sont décisifs dans son style d'apprentissage. Or, selon Norman (2013), pour qu'un utilisateur perçoive l'utilité d'un instrument et, dans notre cas, la possibilité de « mettre ses élèves en autonomie », celui-ci doit intégrer des signifiants reconnaissables et répondre à un besoin existant. En d'autres termes, l'intégration de ces signifiants dès la conception de l'instrument améliorerait l'expérience de l'utilisateur et faciliterait l'efficience de l'usage de l'environnement (Garrett, 2002 ; Hassenzahl, 2010).

Pour ce faire, trois enseignants (P1, P2, P3) ont collaboré lors de trois journées de coconception, au cours desquelles ils ont choisi de créer un EIAH dédié au calcul littéral, puisqu'il s'agit d'une notion complexe que les élèves ont des difficultés à assimiler. Au total, ils ont créé cinq séances, soit une centaine d'exercices à difficulté progressive, en tenant compte des recommandations du Bulletin officiel spécial no 11 (MENJS, 2015). Ils se sont inspirés de leurs propres pratiques et d'exercices provenant de « Labomep », un site internet largement utilisé par la communauté enseignante qui propose des exerciseurs. Chaque séance a été construite suivant un modèle pyramidal : une séance comptait plusieurs exercices, qui eux-mêmes comptaient dix questions. 


\section{Figure 1}

Captures d'écran illustrant la construction pyramidale des séances

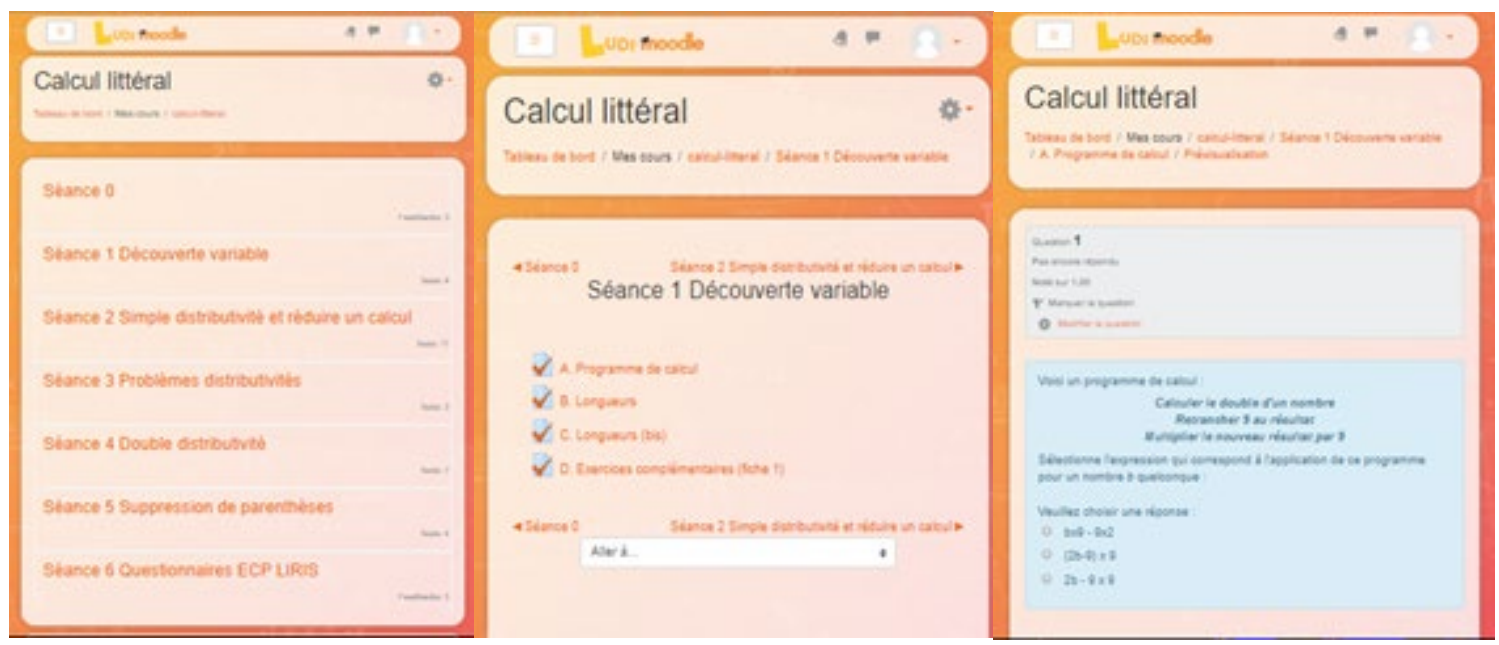

Les enseignants ont également identifié plusieurs besoins devant être satisfaits par l'EIAH :

- Mettre les élèves en autonomie grâce aux rétroactions littérales prévues de manière à ne pas démotiver les élèves, notamment en leur laissant la possibilité de recommencer chaque exercice pour améliorer leur résultat. Lorsqu'ils se trompent, une croix rouge apparaît. Ils ont alors la possibilité de corriger leurs erreurs à la fin des dix questions posées. Pour pouvoir passer à l'exercice suivant, un minimum de $70 \%$ de réussite à l'exercice est requis.

- Leur permettre de s'entraîner sans se soucier du résultat (aucune évaluation subséquente prévue).

- Différencier les apprentissages en adaptant la difficulté des exercices proposés et en proposant des exercices supplémentaires.

- Capter et maintenir l'intérêt en proposant un support « innovant » (exercices réalisés sur tablette numérique).

En ce qui concerne le déroulement des séances d'apprentissage, les enseignants devaient aborder chaque notion en amont de la séance, puis distribuer les tablettes et consacrer au moins 30 minutes à la partie sur l'EIAH. Ils étaient libres cependant d'organiser la séance et la classe comme bon leur semble. 


\section{Participants}

Ce sont 163 élèves, répartis dans 7 classes, qui ont répondu à un questionnaire en prétest et en posttest de l'expérimentation, à 3 semaines d'intervalle, via l'application hébergeant l'exerciseur. La variable « zone de collège » (rural versus citadin) a permis l'étude de l'appartenance socioculturelle des élèves. Il apparaît que $43.6 \%$ des élèves sont en zone urbaine. La proportion de filles et de garçons est respectivement de $47.2 \%$ contre $52.8 \%$. Ces élèves sont répartis comme suit :

\section{Tableau 2}

Répartition de l'échantillon par classe en fonction de l'enseignant, du collège, de la zone et du type de collège

\begin{tabular}{ccccccc}
\hline Classe & $\begin{array}{c}\text { Élèves } \\
n\end{array}$ & $\begin{array}{c}\text { Genre } \\
\text { filles/garçons }\end{array}$ & $\begin{array}{c}\text { Âge } \\
M / E \text { E }\end{array}$ & Enseignant & Collège & Zone \\
\hline $\mathrm{A}$ & 22 & $11 / 11$ & $13.36 / 0.581$ & $\mathrm{P} 3$ & 1 & Rurale \\
\hline $\mathrm{B}$ & 25 & $12 / 13$ & $13.36 / 0.490$ & $\mathrm{P} 2$ & 2 & Rurale \\
\hline $\mathrm{C}$ & 24 & $13 / 11$ & $13.42 / 0.584$ & $\mathrm{P} 1$ & 2 & Rurale \\
\hline $\mathrm{D}$ & 19 & $8 / 11$ & $13.32 / 0.582$ & $\mathrm{P} 4$ & 3 & Citadine \\
\hline $\mathrm{E}$ & 26 & $10 / 16$ & $13.50 / 0.752$ & $\mathrm{P} 5$ & 4 & Citadine \\
\hline $\mathrm{F}^{*}$ & 25 & $11 / 14$ & $13.32 / 0.690$ & $\mathrm{P} 5$ & 4 & Citadine \\
\hline $\mathrm{G}$ & 22 & $12 / 10$ & $13.14 / 0.560$ & $\mathrm{P} 3$ & 1 & Rurale \\
\hline
\end{tabular}

De manière complémentaire, trois entretiens d'explicitation (enseignants $\mathrm{P} 1$, P2 et P3) et quatre captations vidéo (une pour chaque enseignant, sachant que P3 enseigne dans deux classes), portant sur la quatrième séance du calcul littéral nommée « distributivité double », ont été menés auprès des trois enseignants également coconcepteurs de l'EIAH.

\section{Procédures d'analyse}

L'échelle de la motivation à faire des mathématiques. Pour mesurer la motivation d'élèves de 4e année à faire des mathématiques, nous avons adapté l'Échelle de motivation en éducation (Vallerand et al., 1989) à notre population à l'étude et à l'enseignement des mathématiques. Sur la base de ce questionnaire, nous avons retenu une question générale, " pourquoi vas-tu en cours de mathématiques ? », à laquelle les élèves devaient répondre en prétest, puis en posttest (via la tablette numérique), à 28 
items réponses basés sur une échelle de Likert à 5 points $(1=$ ne correspond pas $d u$ tout et 5 = correspond tout à fait ; cf. tableau 11 en annexe). Ces items reprennent les différents types de motivations intrinsèques et extrinsèques identifiés par la TAD (Deci et Ryan, 2000), hormis la motivation extrinsèque à la régulation intégrée rencontrée essentiellement chez l'adulte. Pour plus de simplicité dans la présentation de nos résultats, nous avons donné des acronymes aux différentes variables de la motivation et précisé à quoi correspondait chacune de ces variables dans le cadre de notre échelle de la motivation à faire des mathématiques :

- Motivation intrinsèque à la connaissance $(\mathrm{MICO})$ : apprendre de nouvelles choses

- Motivation intrinsèque à l'accomplissement (MIAC) : relever des défis

- Motivation intrinsèque à la stimulation (MIST) : faire des mathématiques par plaisir

- Motivation extrinsèque à la régulation identifiée (MEID) : faire des mathématiques par utilité (études, passage en 3e, etc.)

- Motivation extrinsèque à la régulation introjectée (MEIN) : pour se sentir compétent

- Motivation extrinsèque à la régulation externe (MERE) : pour obtenir des récompenses (notes, etc.)

- Amotivation (AMOT) : amotivation

\section{La fiabilité de l'échelle de la motivation à faire des mathématiques. Pour} chacune des sous-échelles créées, une analyse factorielle confirmatoire a été réalisée. L'indice KMO (entre .719 et .806), le test de sphéricité $(p=.000<.05)$, la matrice des composantes (entre .511 et .898), le nombre de facteurs (un seul facteur observé) et la variance totale expliquée pour chacune d'elles (entre $55.75 \%$ et $70.30 \%$ ) ont été contrôlés. Nous avons ensuite procédé au regroupement des items par sous-échelles et obtenu pour chacune un alpha de Cronbach satisfaisant (MIAC $\alpha=.777, \operatorname{MICO} \alpha=.783$, MIST $\alpha=.818$, MEID $\alpha=.768$, MEIN $\alpha=.679$, MERE $\alpha=.642$, AMOT $\alpha=.728$ ).

\section{La validation du continuum d'autodétermination à faire des mathématiques.}

Une matrice de corrélations entre les différents types de motivations a été réalisée, révélant des corrélations entre les trois types de motivations intrinsèques oscillant entre 
.734 et .768 , et fortement corrélées à la régulation identifiée $(r=.450, .497$ et .572 pour $p<.01)$, qui elle-même affiche une corrélation avec la régulation introjectée $(\mathrm{r}=.496)$. Enfin, nous constatons que l'amotivation est corrélée négativement à toutes les formes de motivations intrinsèques. Ces corrélations « décroissantes » confirment l'existence d'un continuum d'autodétermination (Deci et Ryan, 1985) qui nous permet d'attester de la validité de notre échelle.

\section{La mesure de la motivation initiale à faire des mathématiques et du gain de} motivation à la suite de l'utilisation de l'EIAH. Du fait de données non paramétriques, notamment de variables ne suivant pas une loi normale (ex., présence de valeurs extrêmes : coefficient d'asymétrie de 1.177 pour l'AMOT et 1.103 pour la MEIN, coefficient de voussure de 2.516 pour la MERE), des tests $H$ de Kruskal-Wallis et $U$ de Mann-Whitney ont été effectués pour déterminer s'il existe des différences significatives de motivation entre les élèves en fonction des variables de sexe, zone de collège et entre classes. Puis, un test des rangs signés de Wilcoxon, comparant les scores obtenus en prétest et en posttest pour chacune des sous-échelles, a permis de mesurer l'effet de l'EIAH mis en place sur la motivation des élèves.

L'évaluation du soutien à l'autonomie. Afin d'évaluer le rôle joué par le soutien à l'autonomie dans la variation de la motivation interclasses, nous avons réalisé des entretiens d'explicitation (Vermersch, 1994) auprès des trois enseignants ayant coconçu l'EIAH, et filmé les séances pour croiser les données issues du verbatim avec celles issues des observations. Nous avons identifié dans leur discours tout ce qui relève des procédures liées à la mise en autonomie, à la structuration de l'enseignement, à l'implication de l'enseignant, à l'adaptation de l'EIAH, et les avons complétées par une observation de l'environnement (disposition des tables) et des interactions de l'enseignant (remédiation, échanges possibles entre pairs).

\section{Résultats}

Pour évaluer l'effet de l'environnement d'apprentissage sur la variation de la motivation des élèves, nous avons dans un premier temps mesuré cette variation au niveau global, puis selon différents niveaux d'analyse : zone du collège, sexe et niveau de motivation 
initiale. Ensuite, nous avons étudié et croisé les résultats motivationnels spécifiques aux classes des trois enseignants (P1, P2 et P3) avec ceux obtenus à la suite des entretiens réalisés avec eux et des observations faites en classe afin de vérifier l'effet du style d'enseignement.

\section{Dépendance de la motivation et des caractéristiques des élèves}

Les résultats du test de Wilcoxon montrent globalement une diminution de la motivation (sauf MERE) et une progression de l'amotivation de l'ensemble des élèves (cf. tableau 3).

\section{Tableau 3}

Variations globales significatives des scores de la motivation

\begin{tabular}{cccccccc}
\hline $\begin{array}{c}\text { Variations de } \\
\text { motivation }\end{array}$ & $\Delta$ AMOT & $\Delta$ MERE & $\Delta$ MEIN & $\Delta$ MEID & $\Delta$ MIST & $\Delta$ MIAC & $\Delta$ MICO \\
\hline$z$ & $2.296^{*}$ & -1.626 & $-3.073^{*}$ & $-2.306^{*}$ & $-2.828^{*}$ & $-2.873^{*}$ & $-4.358^{* *}$ \\
\hline${ }^{*} p<.05,{ }^{* *} p<.001$ & & & & & & &
\end{tabular}

Cette démotivation prend néanmoins des formes différentes selon la zone du collège et le sexe des étudiants. En effet, les élèves de zone urbaine sont plus motivés que ceux de zone rurale à faire des mathématiques pour apprendre de nouvelles choses $(\mathrm{MICO} z=2.577, \mathrm{p}=.01)$ et par plaisir (MIST $\mathrm{z}=2.598, \mathrm{p}=.003)$.

\section{Tableau 4}

Différences de motivations et de variation de la motivation suivant la zone de collège

\begin{tabular}{ll}
\hline Différences entre zones en prétest & Différences entre zones en posttest \\
\hline MICO rurale $<$ urbaine $\left(z=-2.577^{*}\right)$ & MIC rurale $<$ urbaine $\left(z=-2.209^{* *}\right)$ \\
MIST rurale $<$ urbaine $\left(z=-2.598^{* *}\right)$ & MIST rurale $<$ urbaine $\left(z=-2.43^{*}\right)$ \\
\hline${ }^{*} p<.05,{ }^{* *} p<.001$ &
\end{tabular}

Il est aussi remarqué que les garçons sont plus motivés que les filles à faire des mathématiques par plaisir (MIST) et pour relever des défis (MIAC, cf. tableau 5). 


\section{Tableau 5}

Différences de motivations et de variation de la motivation suivant le sexe des élèves

\begin{tabular}{ll}
\hline Différences entre sexes en prétest & Différences entre sexes en posttest \\
\hline MIAC filles $<$ garçons $\left(z=-2.126^{*}\right)$ & MIC filles $<$ garçons $\left(z=-2.115^{*}\right)$ \\
MIST filles $<$ garçons $\left(z=-3.112^{* *}\right)$ & MIST filles $<$ garçons $(z=-2.699 *)$ \\
\hline${ }^{*} p<.05,{ }^{* *} p<.001$ &
\end{tabular}

Des différences sont aussi constatées pour les filles de zone rurale qui seraient plus motivées aux récompenses que celles de zone urbaine $(z=-2.157, p=.031)$ alors que les garçons de zone urbaine seraient plus motivés que ceux de zone rurale à faire des mathématiques par plaisir $(z=-2.789, p=.005)$, pour relever des défis $(z=-2.203$, $p=.028)$ ou pour apprendre de nouvelles choses $(z=-2.096, p=.036)$. Ces premiers résultats montrent des écarts motivationnels différents en fonction des caractéristiques individuelles des élèves et des types de motivations spécifiques.

Il est aussi remarqué que la seule variable de motivation posttest permettant de différencier les élèves (toutes classes et tous collèges confondus) est la MIST $(H=16.803$, $p=.001)$. Nous constatons là aussi des différences en fonction du sexe et de la zone du collège. Les filles éprouvent moins de plaisir que les garçons à faire des mathématiques (MIST). Ce résultat, observé lors du prétest $(z=-3.112, p=.002)$, s'observe également en posttest $(z=-2.075, p=.038)$. De manière encore plus spécifique, les garçons de zone urbaine seraient plus motivés que ceux de zone rurale à faire des mathématiques par plaisir $(z=2.789, p=.005)$. Les élèves « masculins et ruraux » ont vu leur score de motivation à faire des mathématiques par plaisir diminuer $(z=-1.984, p=.035)$, ce qui n'est ni le cas pour les garçons urbains ni pour les filles rurales et urbaines.

Enfin, les différences sur la MIST s'expliquent aussi en fonction du niveau de motivation initiale : les élèves faiblement motivés à faire des mathématiques par plaisir (quartile 1 inférieur à 7 au prétest) ont vu leur motivation augmenter $(z=2.445, p=.014)$, contrairement aux élèves les plus motivés initialement (quartile 3 supérieur à 13). Ce résultat corrobore celui observé, de manière plus générale, entre le niveau de motivation initiale et l'écart de variation : plus un élève se montre initialement peu motivé pour un type de motivation, plus cette même motivation augmente en posttest ; et, inversement, plus l'élève est motivé au départ, plus sa démotivation est grande à la suite de l'expérimentation (cf. tableau 6). 


\section{Tableau 6}

Test de corrélation entre la motivation initiale et la variation de la motivation

\begin{tabular}{lccccccc}
\hline $\begin{array}{c}\text { Coefficient de } \\
\text { corrélation }\end{array}$ & $\Delta$ MICO & $\Delta$ MIAC & $\Delta$ MIST & $\Delta$ MEID & $\Delta$ MEIN & $\Delta$ MERE & $\Delta$ AMOT \\
\hline AMOT initiale & $-.143^{*}$ & -.017 & -.035 & -.017 & -.005 & -.096 & $-.317^{* *}$ \\
MERE initiale & -.041 & -.102 & -.065 & .011 & .022 & $-.297^{* *}$ & -.010 \\
MEIN initiale & .042 & -.022 & -.002 & -.007 & $-.223^{* *}$ & -.085 & .036 \\
MEID initiale & $-.122^{*}$ & -.073 & -.006 & $-.252^{* *}$ & -.082 & -.066 & .055 \\
MIST initiale & .034 & -.067 & $-.260^{* *}$ & -.045 & -.071 & -.058 & .020 \\
MIAC initiale & .030 & $-.229^{* *}$ & -.114 & -.035 & -.073 & -.047 & .025 \\
MICO initiale & -.115 & .014 & -.052 & -.086 & -.059 & -.001 & .054 \\
\hline
\end{tabular}

${ }^{*} p<.05,{ }^{* *} p<.001$

\section{Différents effets selon le style de l'enseignant}

Trois enseignants sont concernés par cette étude, tous situés en zone rurale. En se focalisant sur les quatre classes (A-G, B, C) respectivement des enseignants (P3, P1, P2), si une démotivation est constatée de manière générale, seuls les élèves de la classe $\mathrm{C}$, dirigée par l'enseignant P1, n'ont vu aucune variation motivationnelle significative (cf. tableau 7).

\section{Tableau 7}

Variation de la motivation par classe

\begin{tabular}{lll}
\hline \multicolumn{1}{c}{ Classe } & \multicolumn{1}{c}{ Types de motivations à faire des maths } & \multicolumn{1}{c}{ Résultat } \\
\hline A (enseignant P3) & $\begin{array}{l}\text { Apprendre de nouvelles choses (MICO) } \\
\text { Se sentir compétent (MEIN) }\end{array}$ & $\begin{array}{l}z=-2.242^{*} \\
z=-3.111^{* *}\end{array}$ \\
B (enseignant P2) & Apprendre de nouvelles choses (MICO) & $z=-2.284^{*}$ \\
C (enseignant P1) & & Non significatif \\
G (enseignant P3) & $\begin{array}{l}\text { Apprendre de nouvelles choses (MICO) } \\
\text { Relever des défis (MIAC) }\end{array}$ & $z=-2.236^{*}$ \\
& $\begin{array}{l}\text { Par plaisir (MIST) } \\
\text { Se sentir compétent (MEIN) }\end{array}$ & $z=-3.175^{*}$ \\
& & $z=-2.526^{*}$ \\
\hline
\end{tabular}

$* p<.05, * * p<.001$ 
De plus, il n'est constaté aucune variation motivationnelle significative en fonction du sexe pour les élèves de la classe $\mathrm{C}$ de l'enseignant $\mathrm{P} 1$, contrairement aux trois autres classes (cf. tableau 8)

\section{Tableau 8}

Variation motivationnelle par classe en fonction du sexe

\begin{tabular}{ccl}
\hline Enseignant & Classe & \multicolumn{1}{c}{ Variation de la motivation par sexe et par classe } \\
\hline P1 & C & Pas de différences significatives des filles et des garçons \\
P2 & B & $\begin{array}{l}\text { Pas de différences significatives des filles } \\
\end{array}$ \\
& & $\Delta$ MEID garçons $\left(z=-2.376^{*}\right)$ \\
P3 & A & $\Delta$ MEIN filles $\left(z=-2.687^{*}\right)$ \\
& & $\Delta$ AMOT garçons $\left(z=-1.971^{*}\right)$ \\
& & $\Delta$ MICO garçons $\left(z=-2.561^{*}\right)$ \\
& G & $\Delta$ MIST filles $\left(z=-1.984^{*}\right)$ \\
& & $\Delta$ MIAC filles $\left(z=-2.310^{*}\right)$ \\
& & $\Delta$ MICO filles $\left(z=-2.677^{*}\right)$ \\
& & $\Delta$ MIAC garçons $\left(z=-2.148^{*}\right)$ \\
\end{tabular}

${ }^{*} p<.05,{ }^{* *} p<.001$

Nous notons également des écarts significatifs de variation de la motivation au posttest entre les classes portant uniquement sur la motivation « faire des mathématiques par plaisir » (MIST), au profit des élèves de l'enseignant $\mathrm{P} 1(H=8.709, p=.033)$.

\section{Tableau 9}

Différences de variation motivationnelle par classe et rang moyen

\begin{tabular}{ccccc}
\hline Classe & A & B & C & G \\
\hline Rang moyen & 47.48 & 44.98 & 58.92 & 35.82 \\
\hline
\end{tabular}

Si la motivation « à faire des mathématiques par plaisir » (MIST) semble explicative d'un certain nombre de différences entre les élèves, y compris en ce qui a trait à la variation de la motivation, l'étude par niveaux de motivation initiale (cf. tableau 6) montre un effet positif d'autres variables sur la variation de motivations du même type, illustrant l'aspect multidimensionnel de la motivation à faire des mathématiques des élèves. 
Cependant, les résultats laissent aussi penser que le style de l'enseignant peut avoir un effet sur la variation de la motivation des élèves, en particulier sur la variation de la MIST (plaisir à faire des mathématiques) : l'enseignant P1 a un style davantage « autonomisant » que les deux autres enseignants (cf. tableau 10).

\section{Tableau 10}

\section{Style de l'enseignant en rapport avec l'autonomie}

\begin{tabular}{|c|c|c|c|c|}
\hline Enseignant & Classe & Soutien à l'autonomie & Structuration de l'enseignement & $\begin{array}{c}\text { Implication de } \\
\text { l'enseignant }\end{array}$ \\
\hline $\mathrm{P} 1$ & $\mathrm{C}$ & $\begin{array}{l}\text { Laisse se débrouiller } \\
\text { (demande aux élèves } \\
\text { de ne l'interroger que } \\
\text { pour des problèmes } \\
\text { liés aux mathéma- } \\
\text { tiques) } \\
\text { Échanges entre élèves } \\
\text { réglementés (utilisa- } \\
\text { tion d'un tétraèdre } \\
\text { posé sur la table, indi- } \\
\text { quant qu'ils sollicitent } \\
\text { l'aide d'un pair ou de } \\
\text { l'enseignant) }\end{array}$ & $\begin{array}{l}\text { Anticipe les règles d'écriture de } \\
\text { l'exerciseur } \\
\text { Annote au tableau } \\
\text { Mobilise le vidéoprojecteur } \\
\text { Insère des flèches pour illustrer la } \\
\text { distribution } \\
\text { Met le symbole «-» en rouge } \\
\text { pour limiter le risque d'erreurs } \\
\text { Sélectionne les exercices les plus } \\
\text { simples à réaliser sur tablette } \\
\text { Séance de } 1 \text { h sur tablette } \\
\text { Disposition traditionnelle des } \\
\text { tables : par 2, face au tableau }\end{array}$ & $\begin{array}{l}\text { Fait de la remédia- } \\
\text { tion individuelle } \\
\text { Très peu d'échanges } \\
\text { Adopte une position } \\
\text { en retrait au fond de } \\
\text { la classe } \\
\text { Garde une attitude } \\
\text { neutre face aux diffi- } \\
\text { cultés techniques }\end{array}$ \\
\hline $\mathrm{P} 2$ & B & $\begin{array}{l}\text { Échanges entre élèves } \\
\text { non réglementés }\end{array}$ & $\begin{array}{l}\text { Anticipe les règles d'écriture de } \\
\text { l'exerciseur } \\
\text { Annote au tableau } \\
\text { Mobilise le vidéoprojecteur } \\
\text { Séance de } 1 / 2 \mathrm{~h} \text { sur tablette } \\
\text { Disposition des tables par } 4\end{array}$ & $\begin{array}{l}\text { Fait des remédia- } \\
\text { tions individuelles et } \\
\text { collectives } \\
\text { Circule dans la } \\
\text { classe pendant toute } \\
\text { la partie sur tablette } \\
\text { Garde une attitude } \\
\text { neutre face aux diffi- } \\
\text { cultés techniques }\end{array}$ \\
\hline $\mathrm{P} 3$ & $\begin{array}{l}\mathrm{A} \\
\mathrm{G}\end{array}$ & $\begin{array}{l}\text { Échanges entre élèves } \\
\text { non réglementés }\end{array}$ & $\begin{array}{l}\text { Anticipe les règles d'écriture de } \\
\text { l'exerciseur } \\
\text { Annote au tableau } \\
\text { Mobilise le vidéoprojecteur } \\
\text { Insère des flèches pour illustrer la } \\
\text { distribution } \\
\text { Séance de } 1 \frac{2}{2} \text { heure sur tablette } \\
\text { Disposition des tables par } 4\end{array}$ & $\begin{array}{l}\text { Fait des remédia- } \\
\text { tions individuelles et } \\
\text { collectives } \\
\text { Porte un jugement } \\
\text { sur les problèmes } \\
\text { techniques de l'outil } \\
\text { Circule dans la } \\
\text { classe pendant toute } \\
\text { la partie sur tablette }\end{array}$ \\
\hline
\end{tabular}


L'enseignant P1 est le seul à laisser les élèves « se débrouiller », selon ses mots, et à réglementer les échanges, c'est-à-dire à les autoriser (à condition qu'ils soient liés aux mathématiques) et à les diriger (l'intention de demander une remédiation signalée auparavant grâce à un tétraèdre posé sur la table). Les enseignants $\mathrm{P} 2$ et $\mathrm{P} 3$, au contraire, ont laissé les élèves échanger librement et les interpeller dès qu'une difficulté survenait. P3 trouve par exemple : « la gestion de classe, y a ce facteur dans lequel ils sont un peu isolés avec leur tablette et où ils se coupent un peu du monde et du coup ils ont un petit côté égocentrique quoi, il faut qu'on leur réponde tout de suite et vite ». P2 ajoute quant à lui : « avec tablette, on passe moins de temps au tableau, on passe beaucoup plus de temps dans les rangs, ça se voit dans les vidéos, je ne fais que de marcher, de répondre aux questions ».

Par ailleurs, du point de vue de la structuration de l'enseignement, il a été noté que $\mathrm{P} 1$ est le seul à avoir consacré une heure de cours complète à la partie exercice sur l'EIAH, conservé une disposition des tables traditionnelle (par deux, face au tableau), et sélectionné les exercices les plus simples sur l'EIAH pour ne pas mettre ses élèves en difficulté. À l'inverse, les enseignants $\mathrm{P} 2$ et P3 ont accordé un temps plus court à l'enseignement sur tablette, ont placé les élèves par groupe de quatre et n'ont pas modifié la difficulté sur l'EIAH.

Enfin, des différences d'implication entre les enseignants sont aussi à noter. P1 a adopté une attitude en retrait, limitant ses échanges à de simples remédiations individuelles, sans donner son avis sur les problèmes techniques ou ergonomiques rencontrés par les élèves. P2 et P3 ont au contraire beaucoup interagi avec les élèves de façon individuelle, mais aussi collective, et se sont beaucoup déplacés, nous confiant que les séances avaient été « éreintantes ». Si P2 est également resté neutre face aux difficultés rencontrées, P3, lui, a jugé de façon négative l'outil devant ses élèves.

\section{Discussion}

Les résultats obtenus soulèvent la question de la spécificité de cet EIAH, où la démotivation liée à la régulation externe se caractérise par l'absence d'évaluation prévue à la suite des exercices réalisés, et celle liée à la régulation introjectée par l'absence de possibilité de comparer les résultats entre élèves. La diminution de la motivation à la 
stimulation, quant à elle, porte à croire que l'EIAH n'a pas procuré de plaisir aux élèves, dégradant leur créativité du fait qu'il est impossible d'agir dans et sur cet environnement informatique, le degré de contrôle y étant trop élevé (Amabile, 1996). Ainsi, plus généralement, un EIAH « générique » pose un certain nombre de questionnements quant à ses effets motivationnels (Barata et al., 2017), surtout s'il n'est pas adapté à certaines caractéristiques des élèves, puisque les résultats mettent en évidence l'existence de motivations différentes entre élèves à faire des mathématiques selon la zone de leur collège, leur sexe et leur motivation initiale. Le « niveau de motivation initiale » est ici une variable clairement discriminante du gain motivationnel : les élèves les moins motivés intrinsèquement et extrinsèquement à faire des mathématiques se retrouvent parmi les plus motivés à la suite de l'expérimentation, alors que ceux étant les plus motivés au départ se trouvent davantage démotivés, voire amotivés. Ce résultat corrobore ceux d'autres études (Amabile et al., 1976 ; Lavoué et al., 2018). Il porte à croire que ce type d'EIAH, qui fonctionne par renforcement positif ou négatif, ne serait pas adapté aux élèves intéressés intrinsèquement par les mathématiques, faisant ainsi écho à d'autres études dans le cadre de la TAD (Amabile et al., 1976 ; Deci et al., 1981, 2001 ; McGraw et McCullers, 1979).

Cependant, tous les résultats ne s'expliquent pas dans la seule relation élèvesEIAH puisque des variations de la motivation sont constatées entre classes. Elles s'expliqueraient par le style d'enseignement des enseignants, c'est-à-dire par les conditions créées pour favoriser l'autonomie des élèves, qui concernent à la fois la façon dont l'enseignant structure et s'implique dans l'enseignement. L'enseignant P1, qui a respecté ces trois points en particulier et privilégié une séance d'une heure sur tablette, a su préserver la motivation de ses élèves, contrairement à P3 qui, face aux difficultés, n’a pas démontré une attitude bienveillante ni cherché à remotiver ses élèves. P1 a su, par son style « non contrôlant» — dans le sens entendu par Reeve (2009) et Viau (2009) capter davantage l'intérêt de ses élèves les moins motivés que P2. Par conséquent, bien que l'exploitation de ces résultats soit limitée, puisque seulement trois enseignants ont été interrogés, il apparaît qu'un enseignant qui favorise l'autonomie des élèves influence positivement leur motivation (Chemolli et Gagné, 2014). On perçoit également le lien de dépendance qu'entretient la motivation avec les processus d'autorégulation qui doivent tenir compte des caractéristiques individuelles (Deci et Ryan, 1985 ; Vallerand et al., 
1989) et de la durée d'utilisation de l'environnement numérique (Karsenti et Larose, 2001 ; Li et Ma, 2010).

\section{Conclusion}

Cette recherche montre la nécessité de penser la conception d'un EIAH en favorisant son adaptabilité aux caractéristiques individuelles des élèves, mais également de tenir compte des modalités pédagogiques mises en œuvre par l'enseignant — style d'enseignement favorisant l'autonomie des élèves (enseignement dirigé et non contrôlant) — lorsque celles-ci ne sont pas implémentées dans l'environnement informatique. À la suite de cette première expérimentation, les résultats obtenus, et contrastés, s'expliquent par le fait que tous les enseignants et les élèves ne réagissent pas de la même façon face à l'EIAH (Barata et al., 2017 ; Hanus et Fox, 2015 ; Landers et al., 2017). Du point de vue de la théorie de l'autodétermination, mais aussi des théories sur la ludification, il semble essentiel d'adapter cet EIAH, certes, au profil de joueur de l'élève (Göbel et al., 2010 ; Monterrat, 2015 ; Natkin et al., 2007), mais aussi à un certain nombre de caractéristiques individuelles (Kickmeier-Rust et al., 2014 ; Lavoué et al., 2018 ; Roosta et al., 2016) : niveau de motivation initiale, appartenance socioéconomique et sexe. Plus précisément, une adaptation, la moins statique possible, prenant en compte le profil de l'élève en amont de l'activité, semble nécessaire et devrait être plus efficace qu'un EIAH générique, surtout si l'enseignant n'a pas la possibilité d'agir directement sur l'environnement informatique en le modifiant, en l'adaptant aux élèves, et en mettant en œuvre des modalités dirigées et non contrôlantes vis-à-vis des élèves. 


\section{Annexes}

\section{Tableau 11}

\section{Exemples d'items de l'échelle de motivation à faire des mathématiques}

\begin{tabular}{|c|c|c|}
\hline État motivationnel & Types de motivations & Exemples d'items \\
\hline \multicolumn{3}{|c|}{ Pourquoi vas-tu en cours de Mathématiques ?... } \\
\hline $\begin{array}{c}\text { Amotivation } \\
\text { (AMOT) }\end{array}$ & Aucun & $\begin{array}{l}\text {...je ne sais pas pourquoi j'y vais, j'ai le sentiment de } \\
\text { perdre mon temps. } \\
\text {...j'avais de bonnes raisons au départ d'y aller, mais } \\
\text { aujourd'hui je n'en ai plus. }\end{array}$ \\
\hline \multirow[t]{3}{*}{ Extrinsèque } & $\begin{array}{l}\text { Externe } \\
\text { (MERE) }\end{array}$ & $\begin{array}{l}\text {...parce que je veux avoir de bonnes notes et une } \\
\text { bonne moyenne générale. } \\
\text {...pour pouvoir passer en } 3 \mathrm{e} \text {. }\end{array}$ \\
\hline & $\begin{array}{l}\text { Introjectée } \\
\text { (MEIN) }\end{array}$ & $\begin{array}{l}\text {...pour me prouver que je suis capable de réussir en } \\
\text { mathématiques. } \\
\text {...pour me prouver que je suis compétent.e. }\end{array}$ \\
\hline & $\begin{array}{l}\text { Identifiée } \\
\text { (MEID) }\end{array}$ & $\begin{array}{l}\text {...parce que les mathématiques sont importantes dans } \\
\text { la vie de tous les jours. } \\
\text {..parce que les mathématiques vont me permettre de } \\
\text { travailler dans le domaine que j'aime. }\end{array}$ \\
\hline \multirow[t]{3}{*}{ Intrinsèque } & $\begin{array}{l}\text { à la connaissance } \\
(\mathrm{MICO})\end{array}$ & $\begin{array}{l}\text {...parce que j'éprouve du plaisir à apprendre de nou- } \\
\text { velles choses. } \\
\text {...parce que tout ce qui se rapporte aux mathéma- } \\
\text { tiques m'intéresse. }\end{array}$ \\
\hline & $\begin{array}{l}\text { à l'accomplissement } \\
\text { (MIAC) }\end{array}$ & $\begin{array}{l}\text {...pour le plaisir que je ressens à me sentir capable. } \\
\text {..pour la satisfaction que j'ai de réaliser des activités } \\
\text { scolaires difficiles. }\end{array}$ \\
\hline & $\begin{array}{l}\text { aux sensations } \\
\text { (MIST) }\end{array}$ & $\begin{array}{l}\text {...parce que ça m'amuse de résoudre des problèmes } \\
\text { de mathématiques. } \\
\text {...parce que lorsque je suis en mathématiques, je ne } \\
\text { vois plus le temps passer. }\end{array}$ \\
\hline
\end{tabular}




\section{Références}

Amabile, T. M. (1996). Creativity in context: The social psychology of creativity. Westview.

Amabile, T. M., DeJong, W. et Lepper, M. R. (1976). Effects of externally imposed deadlines on subsequent intrinsic motivation. Journal of Personality and Social Psychology, 34(1), 92-98. https://doi.org/10.1037/0022-3514.34.1.92

Balanskat, A., Blamire, R. et Kefala, S. (2006, 11 décembre). The ICT impact report: A review of studies of ICT impact on schools in Europe. European Schoolnet. https:// oei.org.ar/ibertic/evaluacion/sites/default/files/biblioteca/31 theict impact report in europe.pdf

Barata, G., Gama, S., Jorge, J. et Gonçalves, D. (2017). Studying student differentiation in gamified education: A long-term study. Computers in Human Behavior, 71, 550-585. https://doi.org/10.1016/j.chb.2016.08.049

Benita, M., Shane, N., Elgali, O. et Roth, G. (2017). The important role of the context in which achievement goals are adopted: An experimental test. Motivation and Emotion, 41(2), 180-195. https://doi.org/10.1007/s11031-016-9600-8

Black, A. et Deci, E. L. (2000). The effects of instructors' autonomy support and students' autonomous motivation on learning organic chemistry: A selfdetermination theory perspective. Science Education, 84(6), 740-756. https://doi. org/10.1002/1098-237X(200011)84:6\%3C740::AID-SCE4\%3E3.0.CO;2-3

Blais, M. R., Sabourin, S., Boucher, C. et Vallerand, R. J. (1990). Toward a motivational model of couple happiness. Journal of Personality and Social Psychology, 59(5), 1021-1031. https://doi.org/10.1037/0022-3514.59.5.1021

Boekaerts, M. (2001). Context sensitivity: Activated motivational beliefs, current concerns and emotional arousal. Dans S. Volet et S. Järvelä (dir.), Advances in learning and instruction series. Motivation in learning contexts: Theoretical advances and methodological implications (p. 17-32). Pergamon Press.

Bouffard, T., Mariné, C. et Chouinard, R. (2004). Interdépendance des caractéristiques individuelles et contextuelles dans la motivation à apprendre. Revue des sciences de l'éducation, 30(1), 3-8. https://doi.org/10.7202/011766ar 
Careau, L. et Fournier, A.-L. (2002). La motivation. Disposition à l'étude, (7), 1-21. https://www.aide.ulaval.ca/wp-content/uploads/2015/09/dispositions-a-l-etude-lamotivation.pdf

Chandler, C. et Connell, J. (1987). Children's intrinsic, extrinsic and internalized motivation: A developmental study of children's reasons for liked and disliked behaviours. British Journal of Developmental Psychology, 5(4), 357-365. https:// doi.org/10.1111/j.2044-835X.1987.tb01072.x

Chemolli, E. et Gagné, M. (2014). Evidence against the continuum structure underlying motivation measures derived from self-determination theory. Psychology Assessment, 26(2), 575-585. https://doi.org/10.1037/a0036212

Cheung, A. C. et Slavin, R. E. (2013). The effectiveness of educational technology applications for enhancing mathematics achievement in K-12 classrooms: A meta-analysis. Educational research review, 9, 88-113. https://doi.org/10.1016/j. edurev.2013.01.001

Chirkov, V. I. et Ryan, R. M. (2001). Parent and teacher autonomy-support in Russian and U.S. adolescents: Common effects on well-being and academic motivation. Journal of Cross-Cultural Psychology, 32(5), 618-635. https://doi.org/10.1177/00 $\underline{22022101032005006}$

Chouinard, R., Bergeron, J., Vezeau, C. et Janosz, M. (2010). Motivation et adaptation psychosociale des élèves du secondaire selon la localisation socioéconomique de leur école. Revue des sciences de l'éducation, 36(2), 321-342. https://doi. org/10.7202/044480ar

Ciani, K., Sheldon, K., Hilpert, J. et Easter, M. (2011). Antecedents and trajectories of achievement goals: A self-determination theory perspective. British Journal of Educational Psychology, 81(2), 223-243. https://doi. org/10.1348/000709910X517399

Clot, Y. (2008). La recherche fondamentale de terrain : une troisième voie. Éducation permanente, 4(177), 67-77.

Cosnefroy, L. (2010). Se mettre au travail et y rester : Les tourments de l'autorégulation. Revue Française de Pédagogie, (170), 5-15. https://doi.org/10.4000/rfp.1388 
Damon, W. et Lerner, R. M. (dir.). (2006). Handbook of child psychology: Theoretical models of human development (6e éd.). John Wiley \& Sons.

DeCharms, R. (1968). Personal causation : The internal affective determinants of behavior. Academic Press.

Deci, E. L., Betley, G., Kahle, J., Abrams, L. et Porac, J. (1981). When trying to win: Competition and intrinsic motivation. Personality and social psychology bulletin, 7(1), 79-83. https://doi.org/10.1177/014616728171012

Deci, E. L., Koestner, R. et Ryan, R. M. (2001). Extrinsic rewards and intrinsic motivation in education: Reconsidered once again. Review of Educational Research, 71(1), 1-27. https://doi.org/10.3102/00346543071001001

Deci, E. L. et Ryan, R. M. (1980). The empirical exploration of intrinsic motivational processes. Dans L. Berkowitz (dir.), Advances in experimental social psychology (p. 39-80). Academic Press.

Deci, E. L. et Ryan, R. M. (1985). The general causality orientations scale: Selfdetermination in personality. Journal of Research in Personality, 19(2), 109-134. https://doi.org/10.1016/0092-6566(85)90023-6

Deci, E. L. et Ryan, R. M. (2000). The "what" and "why" of goal pursuits: Human needs and the self-determination of behavior. Psychological Inquiry, 11(4), 227-268. https://doi.org/10.1207/S15327965PLI1104_01

Deci, E. L., Vallerand, R. J., Pelletier, L. G. et Ryan, R. M. (1991). Motivation and education: The self-determination perspective. Educational Psychologist, 26(3/4), 325-346. https://doi.org/10.1080/00461520.1991.9653137

Deterding, S., Dixon, D., Khaled, R. et Nacke, L. (2011, 28-30 septembre). From game design elements to gamefulness: Defining "gamification". Dans A. Lugmayr (resp.), MindTrek '11: Proceedings of the 15th international academic MindTrek conference: Envisioning future media environments, Tempere, Finlande (p. 9-15). Association for computing machinery. https://doi.org/10.1145/2181037.2181040

Fraillon, J., Ainley, J., Schulz, W., Friedman, T. et Gebhardt, E. (2014). Preparing for life in a digital age: The IEA international computer and information literacy study international report. Springer. 
Galand, B., Philippot, P. et Frenay, M. (2006). Structure de buts, relations enseignantsélèves et adaptation scolaire des élèves : Une analyse multi-niveaux. Revue française de pédagogie. Recherches en éducation, (155), 57-72. https://doi. org/10.4000/rfp.225

Garon-Carrier, G., Boivin, M., Guay, F., Kovas, Y., Dionne, G., Lemelin, J.-P., Séguin, J. R., Vitaro, F. et Tremblay, R. E. (2016). Intrinsic motivation and achievement in mathematics in elementary school: A longitudinal investigation of their association. Child Development, 87(1), 165-175. https://doi.org/10.1111/ cdev. 12458

Garrett, J. J. (2002). The elements of user experience: User-centered design for the Web and beyond. New Riders.

Gillet, N., Vallerand, R. J. et Lafrenière, M.-A. K. (2012). Intrinsic and extrinsic school motivation as a function of age : The mediating role of autonomy support. Social Psychology of Education, 15(1), 77-95. https://doi.org/10.1007/ $\underline{\mathrm{s} 11218-011-9170-2}$

Göbel, S., Hardy, S., Wendel, V., Mehm, F. et Steinmetz, R. (2010, 25-29 octobre). Serious games for health : Personalized exergames. Dans A. del Bimbo et S.-F. Chang (resp.), MM '10: Proceedings of the 18th ACM international conference on Multimedia, Firenze, Italy (p. 1663-1666). Association for computing machinery. https://doi.org/10.1145/1873951

Grolnick, W. S. et Ryan, R. M. (1987). Autonomy in children's learning: An experimental and individual difference investigation. Journal of Personality and Social Psychology, 52(5), 890-898. https://doi.org/10.1037/0022-3514.52.5.890

Grolnick, W., Ryan, R. M. et Deci, E. L. (1991). Inner resources for school achievement: Motivational mediators of children's perceptions of their parents. Journal of Educational Psychology, 83(4), 508-517. https://doi. org/10.1037/0022-0663.83.4.508

Hallifax, S., Serna, A., Marty, J.-C., Lavoué, G. et Lavoué, E. (2019, 22-25 octobre). Factors to consider for tailored gamification. Dans J. Arnedo et L. E. Nacke (resp.), CHI PLAY'19 : Proceedings of the Annual Symposium on 
Computer-Human Interaction in Play, Barcelona, Spain (p. 559-572). Association for computing machinery. https://doi.org/10.1145/3311350.3347167

Hanus, M. D. et Fox, J. (2015). Assessing the effects of gamification in the classroom : A longitudinal study on intrinsic motivation, social comparison, satisfaction, effort, and academic performance. Computers \& Education, 80, 152-161. https://doi. org/10.1016/j.compedu.2014.08.019

Harackiewicz, J. M., Manderlink, G. et Sansone, C. (1984). Rewarding pinball wizardry : Effects of evaluation and cue value on intrinsic interest. Journal of Personality and Social Psychology, 47(2), 287-300. https://doi. org/10.1037/0022-3514.47.2.287

Hassenzahl, M. (2010). Experience design: Technology for all the right reasons. Synthesis Lectures on Human-Centered Informatics, 3(1), 1-95. https://doi.org/10.2200/ S00261ED1V01Y201003HCI008

Heider, F. (1982). The psychology of interpersonal relations. Psychology Press.

Jerrim, J. (2016). PISA 2012 : How do results for the paper and computer tests compare? Assessment in Education: Principles, Policy \& Practice, 23(4), 495-518. https:// doi.org/10.1080/0969594X.2016.1147420

Jézégou, A. (2010). Le dispositif GEODE pour évaluer l'ouverture d'un environnement éducatif. Revue de l'Éducation à Distance, 24(2), 83-108. https://edutice. archives-ouvertes.fr/edutice-00489395/document

Karsenti, T. et Fiévez, A. (2014). L'iPad à l'école : De l'adoption à l'innovation. Grand Duc.

Karsenti, T. et Larose, F. (2001). Les TIC... au coeur des pédagogies universitaires : Diversité des enjeux pédagogiques et administratifs. Presses de l'Université du Québec.

Khalili, A. et Shashaani, L. (1994). The effectiveness of computer applications: A metaanalysis. Journal of Research on Computing in Education, 27(1), 48-61. https:// doi.org/10.1080/08886504.1994.10782115

Kickmeier-Rust, M. D., Hillemann, E.-C. et Albert, D. (2014). Gamification and smart feedback: Experiences with a primary school level math app. International 
Journal of Game-Based Learning (IJGBL), 4(3), 35-46. https://doi.org/10.4018/ ijgbl.2014070104

Koivisto, J. et Hamari, J. (2014). Demographic differences in perceived benefits from gamification. Computers in Human Behavior, 35, 179-188. https://doi. org/10.1016/j.chb.2014.03.007

Landers, R. N., Bauer, K. N. et Callan, R. C. (2017). Gamification of task performance with leaderboards: A goal setting experiment. Computers in Human Behavior, 71, 508-515. https://doi.org/10.1016/j.chb.2015.08.008

Lavoué, E., Monterrat, B., Desmarais, M. et George, S. (2018). Adaptive gamification for learning environments. IEEE Transactions on Learning Technologies, 12(1), $16-28$.

Legrain, H. (2003). Motivation à apprendre : Mythe ou réalité ? Points d'étape des recherches en psychologie. L'Harmattan.

Leroux, G., Monteil, J.-M. et Huguet, P. (2017). Apprentissages scolaires et technologies numériques : Une revue critique des méta-analyses. L'Année psychologique, 117(4), 433-465. https://doi.org/10.4074/S0003503317004018

Li, Q. et Ma, X. (2010). A meta-analysis of the effects of computer technology on school students' mathematics learning. Educational Psychology Review, 22(3), 215-243. https://doi.org/10.1007/s10648-010-9125-8

Mageau, G. A. et Vallerand, R. J. (2003). The coach-athlete relationship: A motivational model. Journal of sports science, 21(11), 883-904. https://doi.org/10.1080/02640 $\underline{41031000140374}$

McGraw, K. et McCullers, J. (1979). Evidence of a detrimental effect of extrinsic incentives on breaking a mental set. Journal of Experimental Social Psychology, 15(3), 285-294. https://doi.org/10.1016/0022-1031(79)90039-8

Michko, G. M. (2008, 22-25 octobre). Meta-analysis of effectiveness of technology use in undergraduate engineering education [Conférence]. Dans 38th Annual Frontiers in Education Conference, Saratoga Springs, New York (p. S1A-1S1A-6). IEEE. https://doi.org/10.1109/FIE.2008.4720455 
Ministère de l'Éducation nationale, de la Jeunesse et des Sports (MENJS). (2015, 26 novembre). Programmes pour les cycles 2, 3, 4. Bulletin officiel spécial, (11). https://www.education.gouv.fr/pid285/bulletin_officiel.html?pid bo $=33400$

Miserandino, M. (1996). Children who do well in school : Individual differences in perceived competence and autonomy in above-average children. Journal of Educational Psychology, 88(2), 203-214. https://doi.apa.org/ doi/10.1037/0022-0663.88.2.203

Monterrat, B. (2015). Un système de ludification adaptative d'environnements d'apprentissage fondé sur les profils de joueur des apprenants [Thèse de doctorat, INSA de Lyon]. TEL archives ouvertes. https://tel.archives-ouvertes.fr/ $\underline{\text { tel-01255382 }}$

Morie, M. W. et Goore, B. T. (2019, 29-30 novembre). Adaptability of learning games based on learner profiles in the context of autonomous training. Dans G. Mendy, S. Ouya, I. Dioum et O. Thiaré (dir.), AFRICOMM '2018 : Proceeding of the 10th EAI international conference: e-Infrastructure and e-services for developing countries, Dakar, Senegal (p. 284-293). https://doi. org/10.1007/978-3-030-16042-5_25

Natkin, S., Yan, C., Jumpertz, S. et Marquet. B. (2007, 24-28 septembre). Creating Multiplayer Ubiquitous Games using an adaptive narration model based on a user's model. Dans DiGRA '07: Proceedings of the 2007 DiGRA international conference: Situated play, Tokyo, Japan (p. 431-439). Digital Games Research Association. http://www.digra.org/wp-content/uploads/digitallibrary/07311.31560.pdf

Nix, G. A., Ryan, R. M., Manly, J. B. et Deci, E. L. (1999). Revitalization through selfregulation: The effects of autonomous and controlled motivation on happiness and vitality. Journal of Experimental Social Psychology, 35(3), 266-284. https://doi. org/10.1006/jesp.1999.1382

Norman, D. (1988). The psychology of everyday things. Basic Books.

Norman, D. (2013). The design of everyday things (éd. révisée et augmentée). Basic Books. 
Pelletier, L. et Vallerand, R. (1996). Supervisors' beliefs and subordinates' intrinsic motivation: A behavioral confirmation analysis. Journal of Personality and Social Psychology, 71(2), 331-340. https://doi.org/10.1037/0022-3514.71.2.331

Puentedura, R. R. (2014, 29 juin). Learning, technology, and the SAMR model : Goals, processes, and practice. Ruben R. Puentedura's Weblog. http://www.hippasus. com/rrpweblog/archives/000127.html

Rabardel, P. (1995). Les hommes et les technologies : Approche cognitive des instruments contemporains. Armand Colin.

Reeve, J. (2009). Why teachers adopt a controlling motivating style toward students and how they can become more autonomy supportive. Educational psychologist, 44(3), 159-175. https://doi.org/10.1080/00461520903028990

Reis, H. T., Sheldon, K. M., Gable, S. L., Roscoe, J. et Ryan, R. M. (2000). Daily wellbeing: The role of autonomy, competence, and relatedness. Personality and Social Psychology Bulletin, 26(4), 419-435. https://doi.org/10.1177/0146167200266002

Roosta, F., Taghiyareh, F. et Mosharraf, M. (2016, 27-28 septembre). Personalization of gamification-elements in an e-learning environment based on learners' motivation. Dans IST '2016 : Proceedings of the 8th International Symposium on Telecommunications, Tehran, Iran (p. 637-642). https://doi.org/10.1109/ ISTEL.2016.7881899

Roth, G., Assor, A., Kanat-Maymon, Y. et Kaplan, H. (2007). Autonomous motivation for teaching: How self-determined teaching may lead to self-determined learning. Journal of educational psychology, 99(4), 761-774. https://doi. org/10.1037/0022-0663.99.4.761

Ryan, R. M. et Connell, J. P. (1989). Perceived locus of causality and internalization: Examining reasons for acting in two domains. Journal of Personality and Social Psychology, 57(5), 749-761. https://doi.org/10.1037/0022-3514.57.5.749

Ryan, R. M. et Deci, E. L. (2000). Self-determination theory and the facilitation of intrinsic motivation, social development, and well-being. American Psychologist, 55(1), 68-78. https://doi.org/10.1037/0003-066X.55.1.68 
Ryan, R. M. et Deci, E. L. (2002). Overview of self-determination theory: An organismicdialectical perspective. Dans E. L. Deci et R. M. Ryan (dir.), Handbook of selfdetermination research (p. 3-33). University of Rochester Press.

Ryan, R. M. et Grolnick, W. (1986). Origins and pawns in the classroom: Self-report and projective assessments of individual differences in children's perceptions. Journal of Personality and Social Psychology, 50(3), 550-558. https://doi. org/10.1037/0022-3514.50.3.550

Ryan, R. M., Mims, V. et Koestner, R. (1983). Relation of reward contingency and interpersonal context to intrinsic motivation: A review and test using cognitive evaluation theory. Journal of Personality and Social Psychology, 45(4), 736-750. https://doi.org/10.1037/0022-3514.45.4.736

Sarrazin, P., Tessier, D. et Trouilloud, D. (2006). Climat motivationnel instauré par l'enseignant et implication des élèves en classe : L'état des recherches. Revue française de pédagogie. Recherches en éducation, (157), 147-177. https://doi. org/10.4000/rfp.463

Souchal, C. et Toczek, M.-C. (2010). Buts de réussite, conceptions de l'intelligence, différences de performances liées à l'appartenance socio-économique des élèves : De nouvelles hypothèses explicatives ? Les Sciences de l'éducation - Pour l'Ère nouvelle, 43(1), 13-35. https://doi.org/10.3917/lsdle.431.0013

Supper, W., Guay, F., Falardeau, É., Karsenti, T. et Pelletier, G. (2019). La fréquence d'utilisation de la tablette numérique à des fins pédagogiques et le rendement scolaire des élèves : Le rôle médiateur de la motivation autodéterminée. Revue canadienne de l'éducation, 42(2), 517-552. https://journals.sfu.ca/cje/index.php/ cje-rce/article/view/3685

Tamim, R. M., Bernard, R. M., Borokhovski, E., Abrami, P. C. et Schmid, R. F. (2011). What forty years of research says about the impact of technology on learning: A second-order meta-analysis and validation study. Review of Educational Research, 81(1), 4-28. https://doi.org/10.3102/0034654310393361

Tchounikine, P. et Tricot, A. (2011). Environnements informatiques et apprentissages humains. Dans C. Garbay et D. Kayser (dir.), Informatique et sciences cognitives: influences ou confluence? (p. 167-200). Maison des Sciences de l'Homme. 
Vallerand, R. J. (1997). Toward a hierarchical model of intrinsic and extrinsic motivation. Dans M. P. Zanna (dir.), Advances in experimental social psychology (vol. 29, p. 271-360). Academic Press. https://doi.org/10.1016/S0065-2601(08)60019-2

Vallerand, R. J. (2007). A hierarchical model of intrinsic and extrinsic motivation for sport and physical activity. Dans M. S. Hagger et N. L. D. Chatzisarantis (dir.), Intrinsic motivation and self-determination in exercise and sport (p. 255-279, 356-363). Human Kinetics.

Vallerand, R. J. et Blais, M. (1987). Vers une conceptualisation tripartite de la MI : La MI à la connaissance, à l'accomplissement et aux sensations [Manuscrit inédit]. Laboratoire de psychologie sociale, Université du Québec à Montréal.

Vallerand, R. J., Blais, M. R., Brière, N. M. et Pelletier, L. G. (1989). Construction et validation de l'échelle de motivation en éducation (EME). Revue canadienne des sciences du comportement, 21(3), 323-349. https://doi.org/10.1037/h0079855

Vallerand, R. J. et Reid, G. (1984). On the causal effects of perceived competence on intrinsic motivation : A test of cognitive evaluation theory. Journal of Sport Psychology, 6(1), 94-102.

Vansteenkiste, M., Smeets, S., Soenens, B., Lens, W., Matos, L. et Deci, E. L. (2010). Autonomous and controlled regulation of performance-approach goals : Their relations to perfectionism and educational outcomes. Motivation and Emotion, 34(4), 333-353. https://doi.org/10.1007/s11031-010-9188-3

Vermersch, P. (1994). L'entretien d'explicitation en formation initiale et en formation continue. ESF.

Viau, R. (2009). La motivation en contexte scolaire (2e édition). De Boeck Supérieur.

Zuckerman, M., Porac, J., Lathin, D. et Deci, E. L. (1978). On the importance of selfdetermination for intrinsically motivated behavior. Personality and Social Psychology Bulletin, 4(3), 443-446. https://doi.org/10.1177/014616727800400317 